This validates both the solution of the scalar wave equation and its vectorial correction. Furthermore, a vectorial correction need only be applied to accurate solutions of the scalar wave equation such as in Reference 4, which is partly analytic or the various finite difference solutions reviewed in Reference

Acknowledgments: We are very grateful to P. N. Robson for his support and encouragement. P. C. Kendall acknowledges, with thanks, the support of the Wolfson Foundation and $M$ A. Matin the support of the Commonwealth Scholarship Commission in the United Kingdom.

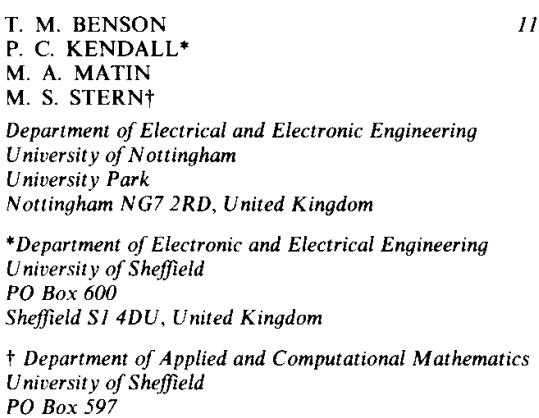

1 ramaswamy, v.: 'Strip loaded film waveguide', Bell Syst. Tech. J., 1974, 53, pp. 697-704

2 BURKE, S. v.: 'Spectral index method applied to coupled rib waveguides', Electron. Lett., 1989, 25, (9), pp. 605-606

3 RAhMAN, B. M. A., and DAVIES, J. B.: 'Vector-H finite element solution of $\mathrm{GaAs} / \mathrm{GaAlAs}$ rib waveguides', IEE Proc. $J, 1985,132$, pp. 349-353

4 MCilroy, P. W. A., STERn, M. S., and Kendall, P. C.: 'Fast and accurate method for calculation of polarised modes in semiconductor rib waveguides', Electron. Lett., 1989, 25, (23), pp. 1586

5 ROBERTSON, M. J., RITCHIE, S., and DAYAN, P.: 'Semiconductor rib waveguide: analysis of optical propagation in rib structures and directional couplers', IEE Proc. J, 1985, 132, pp. 336-342

6 BENSON, T. M., KENDALL, P. C., STERN, M. S., and QUINNEY, D. A.: 'NeW results for rib waveguide propagation constants', IEE Proc. $J$ 1989, 136, (2), pp. 97-102

7 SNYDER, A. W., and LOVE, J. D.: 'Optical waveguide theory' (Chapman and Hall, 1983), pp. 6-9

8 HUANG, w., and HAUS, H. A.: 'A simple variational approach to optical rib waveguides', IEEE J. Lightwave Technol., 1991, 9, pp. optical rib waveguides', IEEE J. Lightwave Technol., 1991, 9, pp 56-61

9 STERN, M. S.: 'Semi-vectorial polarised finite difference method for optical waveguides with arbitrary index profiles', IEE Proc. J, 1988,135 , pp. 56-63

10 STERN, M. S.: 'Rayleigh quotient solution of semivectorial field problems for optical waveguides with arbitrary index profiles', IEE Proc. J, 1991, 138, pp. 185-190

\section{ORIGIN OF ERROR IN CALCULATION OF DISPERSION OF OPTICAL SINGLEMODE FIBRES USING NUMERICAL APPROACH}

Indexing terms: Errors, Optical fibres, Numerical methods

The origin of errors in the calculation of dispersion of optical fibres using an approach presented by Sharma et ol is found fibres using an approach presented by Sharma et al. is found. Such errors are due to the strongly-guided characteristics of
dispersion-flattened and dispersion-shifted optical fibres. dispersion-flattened and dispersion-shifted optical fibres, fibres with weakly-guided field patterns.

Introduction: It is known that various approaches have been adopted to estimate the dispersion of singlemode fibres. Most of the methods have presented sufficient accuracy, but because some of them are used only to handle simple cases such as $\alpha$ power and step index profiles, we found that they may not be able to solve the dispersion to satisfactory accuracy when index profiles become more complicated, such as with doublecladding (W-type) and dispersion-shifted (triangle + ring) fibres. The purpose of this Letter is to explain why one of those approaches presented by Sharma et al. ${ }^{1}$ cannot be applied equally well in more complicated index profile cases. The main reason is the sharper slope of field patterns which is characteristic of newly-invented fibres but not found in conventional ones. This feature will cause errors when solving $b$, $b^{\prime}$, and $b^{\prime \prime}$, and due to the sensitivity of accuracy to errors in these terms, more serious errors will found in the final result. It is also implied that this finding may be able to explain the source of errors when other earlier presented approaches are examined for their accuracy in dealing with newly-presented cases and is found that it is less than expected.

Formulation: We restate the algorithm mentioned in Reference 1 briefly for convenient discussion later. For an optical fibre with refractive index profile given by

$$
\begin{aligned}
n^{2}(R) & =n_{1}^{2}-\left(n_{1}^{2}-n_{2}^{2}\right) \delta f(R) \quad \text { in the core region } \\
& =n_{2}^{2} \quad \text { in the cladding region }
\end{aligned}
$$

if we define $G(R)=(1 / \psi)(d \psi / d R)$, where $\psi$ is the wave field, through Riccati transformation we obtain

$$
\frac{d G}{d R}=v^{2} \delta f(R)-v^{2}(1-b)-\frac{G}{R}-G^{2}
$$

with boundary condition

$$
\begin{aligned}
& G(R=0)=0 \\
& G(R=1)=-\frac{w K_{1}(w)}{K_{0}(w)}
\end{aligned}
$$

By differentiating eqns. 1-3, we can obtain another two equations and their corresponding boundary conditions, and then the system boundary conditions at $R=0$. The numerical steps for solving the equations are summarised as follows:

(1) The transcendental equation (eqn. 3) is solved to obtain $b$, with step size $h=1 / N$. With $b$ known, $G(R)$ is solved through eqn. 1 with step size $h=1 / 4 N$.

(2) The transcendental equation obtained from differentiating eqn. 1 is solved to obtain $b^{\prime}$, with step size $h=1 / N$. With $b^{\prime}$ known, $G^{\prime}(R)$ is solved with step size $h=1 / 2 N$.

(3) The equation and initial conditions obtained from differentiating those of eqn. 3 are solved to obtain $b^{\prime \prime}$, with size $h=1 / N$. By the following equation we can obtain the dispersion $s$ :

$$
\begin{aligned}
s=-\frac{\lambda}{c n_{e}}\left\{(1-b) v_{2}\right. & +b v_{1}+2 \dot{b} \phi \\
& \left.+\frac{1}{2} \ddot{b} \theta-\frac{1}{n_{e}^{2}}\left(n_{2} \dot{n}_{2}+b \phi+\frac{1}{2} \dot{b} \theta\right)^{2}\right\}
\end{aligned}
$$

This method cannot perform equally well when the magnitude of wave field changes greatly in newly-invented fibres. We can understand why by checking the steps mentioned above. Take, for example, step 2 through which $G^{\prime}(R)$ is obtained. As the authors suggested, the Runge-Kutta method is adopted. If

$$
x G_{1}^{\prime}\left(R, G, G^{\prime}, b^{\prime}\right)=-2 G G^{\prime}-\frac{G^{\prime}}{R}-2 v(1-b)+v^{2} b^{\prime}
$$

The Runge-Kutta method then gives

$$
G^{\prime}(i+1)=G^{\prime}(i)+\frac{1}{6}\left(k_{1}+2 k_{2}+2 k_{3}+k_{4}\right)
$$

where $k_{i}$ are accumulative terms obtained from $x G_{1}^{\prime}$. The truncation error of the Runge-Kutta method can be stated as ${ }^{2}$

$$
T E=A(h)^{n+1}+O\left[(h)^{n+2}\right]
$$


and from the Richardson extrapolation ${ }^{2}$

$$
\begin{aligned}
& Y_{i+1}-y_{i+1}=A(h)^{n+1} \frac{x_{i+1}-x_{i}}{h} \\
& Y_{i+1}-\tilde{y}_{i+1}=A\left(\frac{h}{2}\right)^{n+1} \frac{x_{i+1}-x_{i}}{h / 2}
\end{aligned}
$$

where $Y_{i+1}$ is the exact solution, $\tilde{y}$ is the solution when the step is half that of $y$; a more accurate estimation of the error can be given as

$$
\begin{aligned}
T E & =Y_{i+1}-y_{i+1} \\
& =A(h)^{n+1} \\
& =\frac{2^{n}\left(\tilde{y}_{i+1}-y_{i+1}\right)}{2^{n}-1} \\
& =\frac{16}{15}\left(\tilde{y}_{i+1}-y_{i+1}\right) \quad \text { when } n=4
\end{aligned}
$$

Take a point at which $G_{1}^{\prime}(i)=G_{2}^{\prime}(i)$, where $G_{1}^{\prime}(i)$ and $G_{2}^{\prime}(i)$ represent solutions of $G^{\prime}$ when steps are taken as $h$ and $h / 2$, respectively. The truncation error will become $T E=\frac{16}{15}\left[G_{2}^{\prime}(i\right.$ $\left.+1)-G_{1}^{\prime}(i+1)\right]$. Because $G_{j}^{\prime}(i+1)=G_{j}^{\prime}(i)+\frac{1}{6}\left(k_{j 1}+2 k_{j 2}\right.$ $\left.+2 k_{j 3}+k_{j 4}\right), j=1,2$, and $G_{1}^{\prime}(i)=G_{2}^{\prime}(i)$, the above expression can be simplified as

$$
\begin{aligned}
T E= & \frac{16}{15} \frac{h}{6}\left\{\left\{G ^ { \prime } ( i ) \left[-2\left(G_{1}(i)+4 G_{1}(i+1)\right.\right.\right.\right. \\
& \left.+G_{1}(i+2)\right)+\left(G_{2}(i)+4 G_{2}(i+1)\right. \\
& \left.\left.\left.+G_{2}(i+2)\right)\right]\right\}+G^{\prime}(i)\left\{2 \left[G_{1}(i+1) k_{11}\right.\right. \\
& \left.+G_{1}(i+1) k_{12}+G_{1}(i+2) k_{13}\right] \\
& +\left[G_{2}(i+1) k_{21}+G_{2}(i+1) k_{22}\right. \\
& \left.+G_{2}(i+2) k_{23}\right]+\frac{1}{R}\left[\left(k_{11}+k_{12}+k_{13}\right)\right. \\
& \left.\left.\left.+\left(k_{21}+k_{22}+k_{23}\right)\right]\right\}\right\}+4 p
\end{aligned}
$$

where

$$
p=\frac{h}{2}\left(-\frac{G^{\prime}(i)}{R}-2 v(1-b)+v^{2} b^{\prime}\right)
$$

Though the expression looks somewhat more complicated, after careful examination we find that the magnitude of difference depends proportionately on the magnitude of $G$. Because $G(R)=(1 / R)(d \psi / d R)$, obviously the magnitude of $G$ is proportional to the slope of the wave field. As in Fig. 1, we find that wavefield changes most greatly in dispersion-flattened and dispersion-shifted fibres, which means the truncation error of this approach will be larger in these two cases. Although the error is small compared to the result obtained from the variation formulation and finite-element method ${ }^{3}$ it can no longer be ignored in the calculation of dispersion. Because $b, b^{\prime}$ and $b^{\prime \prime}$ are solved step by step, the error caused in each step will accumulate. That is exactly why the errors of these three quantities becomes larger and larger, obeying the same order as that of the steps. Moreover, practically it is found that the accuracy of dispersion is very sensitive to changes of the three values; that is, small errors of $b, b^{\prime}$ and $b^{\prime \prime}$ cause error of dispersion to a much larger degree. Besides, round-off error will become large before the truncation error has reduced sufficiently if the number of steps is increased for more accurate results. The corresponding results of error are shown in Fig. 2. In conventional type fibres, the light wave is weakly-guided and accurate results can be obtained with no difficulty, but in strongly-guided cases, much more attention should be paid to finding the optimal number of steps for the most accurate results. Also, such a strongly-guided feature should be noticed whenever the possible reasons for errors in calculating dispersion are sought, and the same conclusion may be made as those we have made here.

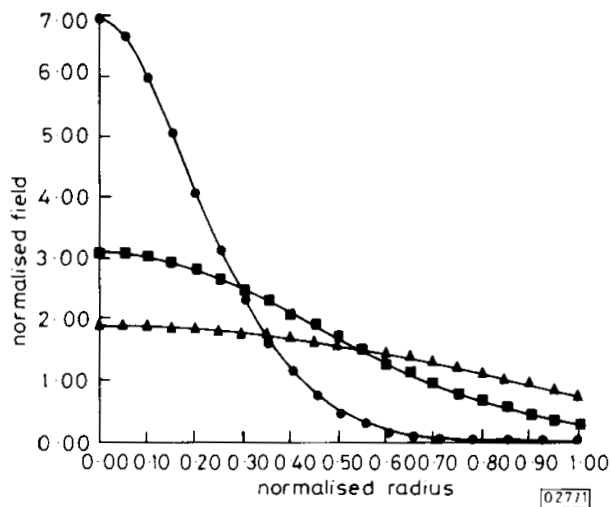

Fig. 1 Normalised field of different core index profile optical fibres

dispersion-shifted

$\alpha$ power $(\alpha=2)$

step index

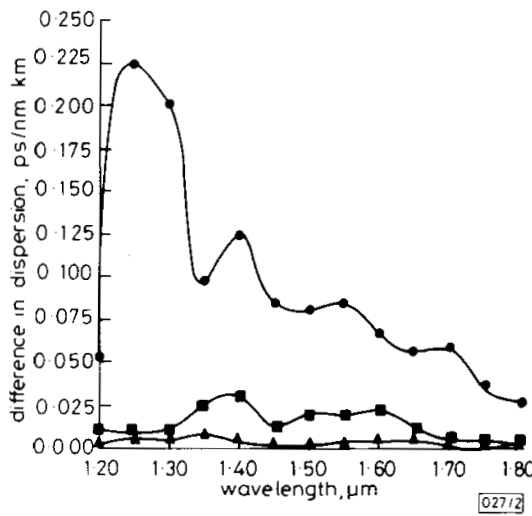

Fig. 2 Error curves of dispersion using method in Reference 1 for different index

dispersion-shifted

$\alpha$ power $(\alpha=$

step index

Conclusion: In this Letter the origin of errors in calculating optical fibre dispersions using the approach in Reference 1 is found. Knowing the origin of such errors, numerical methods other than the Runge-Kutta method can be sought to solve the same equations or the use of the Runge-Kutta approach can be restricted to weakly-guided cases, in which the results are accurate enough, and another method used for stronglyguided cases.

L.-P. YUAN

8th June 1991

Department of Electrical Engineering

National Taiwan University

Taipei, Taiwan, Republic of China

PO Box 23-134

Taipei, Taiwan, Republic of China

\section{References}

1 Sharma, E. K., Sharma, A., and Goyal, I. C.: 'Propagation characteristics of single-mode optical fibers with arbitrary index profiles: a simple numerical approach', IEEE Trans., 1982, MTT-30, pp. $1472-1477$

2 JALURIA, Y.: 'Computer methods for engineering' (Allyn and Bacon, 1988)

3 CHANG, H. C., and LIN, H. Y.: 'Analysis of the propagation characteristics of dispersion-shifted optical fibers using finite-element method'. Dpt. of Electrical Engineering, National Taiwan University, 1989 